\title{
Multifuzzy Cubic Sets and Their Correlation Coefficients for Multicriteria Group Decision-Making
}

\author{
Jun Ye (D), Shigui Du, and Rui Yong \\ School of Civil and Environmental Engineering, Ningbo University, Ningbo 315211, China \\ Correspondence should be addressed to Jun Ye; yejun1@nbu.edu.cn
}

Received 12 January 2021; Revised 28 January 2021; Accepted 1 February 2021; Published 18 February 2021

Academic Editor: Harish Garg

Copyright $\odot 2021$ Jun Ye et al. This is an open access article distributed under the Creative Commons Attribution License, which permits unrestricted use, distribution, and reproduction in any medium, provided the original work is properly cited.

\begin{abstract}
The notion of multifuzzy sets (MFSs) or multi-interval-valued fuzzy sets (MIVFSs) provides a new method to represent some problems with a sequence of the different and/or same fuzzy/interval-valued fuzzy membership values of an element to the set. Then, a fuzzy cubic set (FCS) consists of a certain part (a fuzzy value) and an uncertain part (an interval-valued fuzzy value) but cannot represent hybrid information of both MFS and MIVFS. To adequately depict the opinion of several experts/decisionmakers by using a union/sequence of the different and/or same fuzzy cubic values for an object assessed in group decision-making (GDM) problems, this paper proposes a multifuzzy cubic set (MFCS) notion as the conceptual extension of FCS to express the hybrid information of both MFS and MIVFS in the fuzzy setting of both uncertainty and certainty. Then, we propose three correlation coefficients of MFCSs and then introduce correlation coefficients of MFSs and MIVFSs as special cases of the three correlation coefficients of MFCSs. Further, the multicriteria GDM methods using three weighted correlation coefficients of MFCSs are developed under the environment of MFCSs, which contains the MFS and MIVFS GDM methods. Lastly, these multicriteria GDM methods are applied in an illustrative example on the selection problem of equipment suppliers; then their decision results and comparative analysis indicate that the developed GDM methods are more practicable and effective and reflect that either different correlation coefficients or different information expressions can also impact on the ranking of alternatives. Therefore, this study indicates the main contribution of the multifuzzy cubic information expression, correlation coefficients, and GDM methods in the multifuzzy setting of both uncertainty and certainty.
\end{abstract}

\section{Introduction}

Fuzzy set (FS) [1] is usually depicted by a membership degree in the interval $[0,1]$, where only indicate one occurrence of each element. Hence, FSs have been wildly applied in various areas [2-10] regarding vague and incomplete real problems. As the extension of FSs, Yager [11] and Sebastian and Ramakrishnan [12] presented a fuzzy multiset/bag/multifuzzy set (MFS), which provides a better method to represent the numbers of copies of an element to the set by the different and/or same membership values. Thus, fuzzy multisets/MFSs have been utilized for various applications [13-17]. By extending the fuzzy multiset/MFS to the interval-valued fuzzy set (IVFS) [18], Kreinovich and Sriboonchitta [19] defined the notion of a multi-intervalvalued fuzzy set (MIVFS), where each element can be repeated more than once with the different and/or same interval-valued membership values to adequately describe a union/sequence of interval-valued fuzzy values (IVFVs).

Then, there exist the certainty and uncertainty of human judgments regarding complicated real-world problems. Hence, Jun et al. [20] proposed a notion of a fuzzy cubic set (FCS), which can depict the hybrid fuzzy information of the partial certainty (fuzzy value) and partial uncertainty (IVFV). Afterward, some researchers [21-24] also introduced some theory and applications of FCSs since FCS is a very useful tool for expressing potentially uncertain and certainty information provided by the decision-makers. Based on the hybrid notion of FCS and a hesitant FS [25], some researchers proposed hesitant FCSs and their decisionmaking methods $[26,27]$ due to the hesitancy of human judgments in the setting of FCSs; then some researchers 
presented a cubic hesitant fuzzy set (CHFS) by means of a hybrid form of both an IVFV and several hesitant fuzzy values and after that developed similarity measures of CHFSs for medical diagnosis/assessments $[28,29]$ and decision-making [30]. More recently, $\mathrm{Fu}$ and $\mathrm{Ye}$ [31] proposed the notion of cubic hesitant neutrosophic numbers (CHNNs) by using a hybrid form of both an IVFV and several neutrosophic numbers and then developed the indeterminate parameter-based similarity measure of CHNNs for the risk grade assessment of prostate cancer patients. Next, Ye et al. [32] further proposed a notion of fuzzy credibility cubic numbers (FCCNs) by a hybrid form of both a cubic credibility degree and a fuzzy cubic value (FCV) and then developed two weighted aggregation operators of FCCNs and used them in the decision-making problem of slope design schemes so as to enhance the credibility degree/ level of fuzzy decision-making problems. Based on the mixed form of an intuitionistic fuzzy set (IFS) and an interval-valued IFS (IVIFS), some researchers [33-35] presented cubic intuitionistic fuzzy sets (CIFSs) and their applications in decision-making problems. Although extension forms of various fuzzy information, such as Pythagorean FSs [36], interval-valued Pythagorean FSs [37], and neutrosophic sets [38], were introduced in recent years and applied in GDM problems, they lack corresponding cubic information expression forms.

However, the hesitant FS concept can only express a set of several different cubic membership values of each element to the set in the hesitant environment, but it cannot reflect the number of copies of an element to the set by a union/sequence of the different and/or same membership values so as to lose some useful information in the group decision-making (GDM) process. Generally, the aforementioned various fuzzy cubic concepts cannot also reflect the number of copies of an element to the set by a union/ sequence of the different and/or same FCVs. Then, MFS or MIVFS can reflect a union of the different and/or same fuzzy values or IVFVs of an element to the set, but MFS or MIVFS cannot express the mixed information of both MFS and MIVFS. To suitably describe the union/sequence of the different and/or same FCVs, this study presents the concept of MFCS based on the hybrid information of MFS and MIVFS. The MFCS notion can provide a new expression form to overcome the insufficiency of the hesitant FCS and to extend the notion of FCS. Thus, MFCS can describe the opinions that the several experts or decision-makers propose by using a union/sequence of the different and/or same FCVs in GDM problems. Next, we propose three correlation coefficients of MFCSs and then introduce the correlation coefficients of MFSs and MIVFSs as special cases of the three correlation coefficients of MFCSs. GDM methods using the weighted correlation coefficients of MFCSs (including weighted correlation coefficients of MFSs and MIVFSs) are developed and used in an illustrative example on the selection problem of equipment suppliers. Finally, the comparative analysis of the developed GDM methods and the related methods are investigated to illustrate the practicability and suitability of the developed GDM methods in the setting MFCSs.
In the original study, the main contributions are summarized in the following:

(i) The proposed MFCS can solve the hybrid information expression problem of both MFS and MIVFS and provide an adequate expression form to depict the opinion of several experts/decisionmakers by using a union/sequence of several different and/or the same FCVs in multicriteria GDM problems

(ii) The proposed correlation coefficients of MFCSs can provide effective mathematical models for the multicriteria GDM methods in the environments of MFCSs, MFSs, and MIVFSs

(iii) The developed multifuzzy cubic GDM methods can solve GDM problems under the environment of MFCSs and show their superiority and usability in the setting of MFCSs

For this study, this paper is composed of the following structures. Section 2 introduces some notions of MFSs, MIVFSs, and FCSs. In Section 3, we present the concept of MFCS based on the hybrid information of MFS and MIVFS and define the relations of multifuzzy cubic values (MFCVs). Section 4 proposes three correlation coefficients of MFCSs and then introduces the correlation coefficients of MFSs and MIVFSs as special cases of the three correlation coefficients of MFCSs. Section 5 develops multicriteria GDM methods using the proposed weighted correlation coefficients of MFCSs in the MFCS setting. In Section 6, the developed GDM methods are used for an illustrative example on the selection problem of equipment suppliers, and then the comparative analysis of the developed multicriteria GDM methods and the related methods are investigated to illustrate the practicability and suitability of the developed GDM methods in the setting of MFCSs. Conclusions and further work are included in Section 7.

\section{Some Notions of MFSs, MIVFSs, and FCSs}

Set $U=\left\{u_{1}, u_{2}, \ldots, u_{n}\right\}$ as a universe set. Then, a MFS $S$ on $U$ is defined as the following form $[11,12]$ :

$$
S=\left\{\left\langle u_{j}, M_{S}\left(u_{j}\right)\right\rangle \mid u_{j} \in U\right\},
$$

where $M_{S}\left(u_{j}\right)$ is the membership sequence denoted by $\left(m_{S 1}\left(u_{j}\right), m_{S 2}\left(u_{j}\right), \ldots, m_{S q(j)}\left(u_{j}\right)\right)$ for $m_{S k}\left(u_{j}\right) \in[0,1]$ $(k=1,2, \ldots, q(j) ; j=1,2, \ldots, n)$ and $u_{j} \in U$, which is named a MFS/FMS. In the MFS $S$, each element $u_{j}$ in $U$ may occur more than once with the different and/or same membership values.

To depict the fuzzy uncertain information in real-world problems, Kreinovich and Sriboonchitta [19] put forward a MIVFS $T$ on a universe set $U=\left\{u_{1}, u_{2}, \ldots, u_{n}\right\}$ and defined the following form:

$$
T=\left\{\left\langle u_{j}, M_{T}\left(u_{j}\right)\right\rangle \mid u_{j} \in U\right\},
$$

where $M_{T}\left(u_{j}\right)$ is a union/sequence of the interval-valued membership values defined by $\left(m_{T 1}\left(u_{j}\right), m_{T 2}\left(u_{j}\right), \ldots, m_{T q(j)}\right.$ $\left.\left(u_{j}\right)\right)$ for $m_{T k}\left(u_{j}\right) \subseteq[0,1](k=1,2, \ldots, q(j) ; j=1,2, \ldots, n)$ and 
$u_{j} \in U$, which is named a MIVFS. In the MIVFS $T$, each element $u_{j}$ in $U$ may occur more than once with the different and/or same interval-valued membership values.

To represent the fuzzy hybrid information of the uncertainty and the certainty in real-world problems, Jun et al. [20] put forward a FCS on a universe set $U=\left\{u_{1}, u_{2}, \ldots, u_{n}\right\}$, which is denoted by

$$
C=\left\{\left\langle u_{j}, A_{C}\left(u_{j}\right), \alpha_{C}\left(u_{j}\right)\right\rangle \mid u_{j} \in U\right\},
$$

where $A_{C}\left(u_{j}\right): U \longrightarrow[0,1]$ and $\alpha_{C}\left(u_{j}\right): U \longrightarrow[0,1]$ are an IVFV and a fuzzy value, respectively, such that $A_{C}\left(u_{j}\right)=$ $\left[a\left(u_{j}\right), b\left(u_{j}\right)\right] \subseteq[0,1]$ and $\alpha_{C}\left(u_{j}\right) \in[0,1]$ for $u_{j} \in U(j=1,2, \ldots$, $n)$. In the FCS $C$, each element $u_{j}$ in $U$ only occurs once with the hybrid information of both an interval-valued membership value and a single-valued membership value.

For the simplified expression, each element $<u_{j}, A_{C}\left(u_{j}\right)$, $\alpha_{C}\left(u_{j}\right)>(j=1,2, \ldots, n)$ in $C$ is simply denoted as $c_{j}=<\left[a_{j}, b_{j}\right]$, $\alpha_{j}>$ and named FCV. If $a_{j} \leq \alpha_{j} \leq b_{j}$, then $c_{j}=<\left[a_{j}, b_{j}\right]$, and $\alpha_{j}>$ is named an internal FCV; if $\alpha_{j} \notin\left[a_{j}, b_{j}\right]$, then $c_{j}=<\left[a_{j}, b_{j}\right]$, and $\alpha_{j}>$ is named an external FCV.
For any two FCVs $c_{1 j}=\left\langle\left[a_{1 j}, b_{1 j}\right], \alpha_{1 j}\right\rangle$ and $c_{2 j}=\left\langle\left[a_{2 j}, b_{2 j}\right], \alpha_{2 j}\right\rangle$, their relations are introduced as follows [20]:
(1) $c_{1 j} \supseteq c_{2 j} \Leftrightarrow\left[a_{1 j}, b_{1 j}\right] \supseteq\left[a_{2 j}, b_{2 j}\right]$ and $\alpha_{1 j} \geq \alpha_{2 j}$
(2) $c_{1 j}=c_{2 j} \Leftrightarrow c_{1 j} \supseteq c_{2 j}$ and $c_{2 j} \supseteq c_{1 j}$, namely, $\left[a_{1 j}, b_{1 j}\right]=\left[a_{2 j}, b_{2 j}\right]$ and $\alpha_{1 j}=\alpha_{2 j}$
(3) $c_{1 j} \cup c_{2 j}=\left\langle\left[a_{1 j} \vee a_{2 j}, b_{1 j} \vee b_{2 j}\right], \alpha_{1 j} \vee \alpha_{2 j}\right\rangle$
(4) $c_{1 j} \cap c_{2 j}=\left\langle\left[a_{1 j} \wedge a_{2 j}, b_{1 j} \wedge b_{2 j}\right], \alpha_{1 j} \wedge \alpha_{2 j}\right\rangle$
(5) $c_{1 j}^{c}=\left\langle\left[1-b_{1 j}, 1-a_{1 j}\right], 1-\alpha_{1 j}\right\rangle$ (complement of $c_{1 j}$ )

\section{Multifuzzy Cubic Sets (MFCSs)}

Based on the hybrid concept of MFS and MIVFS, this section presents the concept of MFCS as an extension of the FCS notion and defines the relations of MFCVs.

Definition 1. Set $U=\left\{u_{1}, u_{2}, \ldots, u_{n}\right\}$ as a universe set. A MFCS $M$ over $U$ is defined by the following mathematical expression:

$$
M=\left\{\left\langle\begin{array}{c}
u_{j},\left(\left[a_{M 1}\left(u_{j}\right), b_{M 1}\left(u_{j}\right)\right], \alpha_{M 1}\left(u_{j}\right)\right), \\
{\left[a_{M 2}\left(u_{j}\right), b_{M 2}\left(u_{j}\right)\right], \alpha_{M 2}\left(u_{j}\right), \ldots,} \\
\left(\left[a_{M q(j)}\left(u_{j}\right), b_{M q(j)}\left(u_{j}\right)\right], \alpha_{M q(j)}\left(u_{j}\right)\right)
\end{array}\right\rangle u_{j} \in U\right\},
$$

where $\left(\left[a_{M 1}\left(u_{j}\right), b_{M 1}\left(u_{j}\right)\right], \alpha_{M 1}\left(u_{j}\right)\right),\left(\left[a_{M 2}\left(u_{j}\right), b_{M 2}\left(u_{j}\right)\right]\right.$, $\left.\alpha_{M 2}\left(u_{j}\right)\right), \ldots,\left(\left[a_{M q(j)}\left(u_{j}\right), b_{M q(j)}\left(u_{j}\right)\right], \alpha_{M q(j)}\left(u_{j}\right)\right)$ is a membership sequence of $q(j)$ copies of an element $u_{j}$ to the set $M$, such that IVFVs $\left[a_{M k}\left(u_{j}\right), b_{M k}\left(u_{j}\right)\right] \subseteq[0,1]$ and fuzzy values $\alpha_{M k}\left(u_{j}\right) \in[0,1](j=1,2, \ldots, n ; k=1,2, \ldots, q(j))$ for $u_{j} \in \mathrm{U}$. In the MFCS $M$, each element $u_{j}$ may occur more than once with the different and/or same FCVs.

For example, there is a MFCS $M_{1}=\left\{<u_{1},([0.7,0.9], 0.8)\right.$, $([0.7,0.9], 0.8),([0.5,0.7], 0.6)>,<u_{2},([0.7,0.8], 0.7),([0.7$, $0.8], 0.7),([0.6,0.7], 0.6)>\}$ in the universe set $U=\left\{u_{1}, u_{2}\right\}$.

In particular, when $q(j)=1(j=1,2, \ldots, n)$ in $M$, the MFCS $M$ is reduced to FCS. If $\alpha_{j k} \in\left[a_{j k}, b_{j k}\right](j=1,2, \ldots, n$; $k=1,2, \ldots, q(j)), M$ is named the internal MFCS; and if $\alpha_{j k} \notin\left[a_{j k}, b_{j k}\right](j=1,2, \ldots, n ; k=1,2, \ldots, q(j)), M$ is named the external MFCS.

Each MFCV $\left\langle u_{j},\left(\left[a_{M 1}\left(u_{j}\right), b_{M 1}\left(u_{j}\right)\right], \alpha_{M 1}\left(u_{j}\right)\right),\left(\left[a_{M 2}\right.\right.\right.$ $\left.\left.\left(u_{j}\right), b_{M 2}\left(u_{j}\right)\right], \alpha_{M 2}\left(u_{j}\right)\right), \ldots,\left(\left[a_{M q(j)}\left(u_{j}\right), b_{M q(j)}\left(u_{j}\right)\right], \alpha_{q(j)}\right.$ $\left.\left.\left(u_{j}\right)\right)\right\rangle(j=1,2, \ldots, n)$ in $M$ is simply denoted as $m_{j}=\left\langle\left(\left[a_{j 1}\right.\right.\right.$, $\left.\left.\left.b_{j 1}\right], \alpha_{j 1}\right),\left(\left[a_{j 2}, b_{j 2}\right], \alpha_{j 2}\right), \ldots,\left(\left[a_{j q(j)}, b_{j q(j)}\right], \alpha_{j q}(j)\right)\right\rangle$.

Definition 2. Set any two MFCVs as $m_{1 j}=\left\langle\left(\left[a_{1 j 1}, b_{1 j 1}\right]\right.\right.$, $\left.\left.\alpha_{1 j 1}\right),\left(\left[a_{1 j 2}, b_{1 j 2}\right], \alpha_{1 j 2}\right), \ldots,\left(\left[a_{1 j q(j)}, b_{1 j q(j)}\right], \alpha_{1 j q(j)}\right)\right\rangle$ and $m_{2 j}=\left\langle\left(\left[a_{2 j 1}, b_{2 j 1}\right], \alpha_{2 j 1}\right), \quad\left(\left[a_{2 j 2}, b_{2 j 2}\right], \alpha_{2 j 2}\right), \ldots,\left(\left[a_{2 j q(j)}\right)\right.\right.$, $\left.\left.\left.b_{2 j q(j)}\right], \alpha_{2 j q(j)}\right)\right\rangle(j=1,2, \ldots, n)$. Then, their relations are defined as follows:

(1) $m_{1 j} \subseteq m_{2 j} \Leftrightarrow\left[a_{1 j k}, b_{1 j k}\right] \subseteq\left[a_{2 j k}, b_{2 j k}\right]$ and $\alpha_{1 j k} \leq \alpha_{2 j k}$ $(k=1,2, \ldots, q(j))$;
(2) $m_{1 j}=m_{2 j} \Leftrightarrow m_{1 j} \supseteq m_{2 j}$ and $m_{2 j} \supseteq m_{1 j}$, namely, $\left[a_{1 j k}, b_{1 j k}\right]=\left[a_{2 j k}, b_{2 j k}\right]$ and $\alpha_{1 j k}=\alpha_{2 j k}(k=1,2, \ldots$, $q(j))$

(3) $m_{1 j} \cup m_{2 j}=\left\langle\left[\left[a_{1 j 1} \vee a_{2 j 1}, b_{1 j 1} \vee b_{2 j 1}\right], \alpha_{1 j 1} \vee \alpha_{2 j 1}\right]\right.$, $\left(\left[a_{1 j 2} \vee a_{2 j 2}, b_{1 j 2} \vee b_{2 j 2}\right], \alpha_{1 j 2} \vee \alpha_{2 j 2}\right), \ldots,\left(\left[a_{1 j q(j)} \vee\right.\right.$ $\left.\left.\left.a_{2 j q(j)}, b_{1 j q(j)} \vee b_{2 j q(j)}\right], \alpha_{1 j q(j)} \vee \alpha_{2 j q(j)}\right)\right\rangle$

(4) $m_{1 j} \cap m_{2 j}=\left\langle\left(\left[a_{1 j 1} \wedge a_{2 j 1}, b_{1 j 1} \wedge b_{2 j 1}\right], \alpha_{1 j 1} \wedge \alpha_{2 j 1}\right)\right.$, $\left(\left[a_{1 j 2} \wedge a_{2 j 2}, b_{1 j 2} \wedge b_{2 j 2}\right], \alpha_{1 j 2} \wedge \alpha_{2 j 2}\right), \ldots,\left(\left[a_{1 j q(j)} \wedge\right.\right.$ $\left.\left.\left.a_{2 j q(j)}, b_{1 j q(j)} \wedge b_{2 j q(j)}\right], \alpha_{1 j q(j)} \wedge \alpha_{2 j q(j)}\right)\right\rangle$

(5) $m_{1 j}^{c}=\left\langle\left(\left[1-b_{1 j 1}, 1-a_{1 j 1}\right], 1-\alpha_{1 j 1}\right),\left(\left[1-b_{1 j 2}, 1-a_{1 j 2}\right]\right.\right.$, $\left.\left.1-\alpha_{1 j 2}\right), \ldots,\left[1-b_{1 j q(j)}, 1-a_{1 j q(j)}\right], 1-\alpha_{1 j q(j)}\right\rangle \quad(\mathrm{com}-$ plement of $m_{1 j}$ )

\section{Correlation Coefficients of MFCSs}

Three correlation coefficients of MFCSs and their special cases are proposed in this section under environments of MFCSs, MFSs, and MIVFSs.

Definition 3. Set $M_{1}=\left\{m_{11}, m_{12}, \ldots, m_{1 n}\right\}$ and $M_{2}=\left\{m_{21}\right.$, $\left.m_{22}, \ldots, m_{2 n}\right\}$ as two MFCSs, where $m_{1 j}=<\left(\left[a_{1 j 1}, a_{1 j 1}\right], \alpha_{1 j 1}\right)$, $\left(\left[\begin{array}{lllll}a_{1 j 2}, & a_{1 j 2}\end{array}\right], \alpha_{1 j 2}\right), \ldots, \quad\left(\left[a_{1 j q(j)}, a_{1 j q(j)}\right], \quad \alpha_{1 j q(j)}\right)>\quad$ and $m_{2 j}=<\left(\left[a_{2 j 1}, a_{2 j 1}\right], \alpha_{2 j 1}\right),\left(\left[a_{2 j 2}, a_{2 j 2}\right], \alpha_{2 j 2}\right), \ldots,\left(\left[a_{2 j q(j)}, a_{2 j q(j)}\right]\right.$, $\left.\alpha_{2 j q(j)}\right)>(j=1,2, \ldots, n)$ are MFCVs. Then, three correlation coefficients between $M_{1}$ and $M_{2}$ are proposed as follows: 


$$
\begin{aligned}
& C_{1}\left(M_{1}, M_{2}\right)=\frac{N\left(M_{1}, M_{2}\right)}{\sqrt{N\left(M_{1}, M_{1}\right)} \sqrt{N\left(M_{2}, M_{2}\right)}}=\frac{\sum_{j=1}^{n} \sum_{k=1}^{q(j)}\left(a_{1 j k} a_{2 j k}+b_{1 j k} b_{2 j k}+\alpha_{1 j k} \alpha_{2 j k}\right)}{\sqrt{\sum_{j=1}^{n} \sum_{k=1}^{q(j)}\left(a_{1 j k}^{2}+b_{1 j k}^{2}+\alpha_{1 j k}^{2}\right)} \sqrt{\sum_{j=1}^{n} \sum_{k=1}^{q(j)}\left(a_{2 j k}^{2}+b_{2 j k}^{2}+\alpha_{2 j k}^{2}\right)}}, \\
& C_{2}\left(M_{1}, M_{2}\right)=\frac{N\left(M_{1}, M_{2}\right)}{\max \left(N\left(M_{1}, M_{1}\right), N\left(M_{2}, M_{2}\right)\right)}=\frac{\sum_{j=1}^{n} \sum_{k=1}^{q(j)}\left(a_{1 j k} a_{2 j k}+b_{1 j k} b_{2 j k}+\alpha_{1 j k} \alpha_{2 j k}\right)}{\max \left(\sum_{j=1}^{n} \sum_{k=1}^{q(j)}\left(a_{1 j k}^{2}+b_{1 j k}^{2}+\alpha_{1 j k}^{2}\right), \sum_{j=1}^{n} \sum_{k=1}^{q(j)}\left(a_{2 j k}^{2}+b_{2 j k}^{2}+\alpha_{2 j k}^{2}\right)\right)}, \\
& C_{3}\left(M_{1}, M_{2}\right)=\frac{\sum_{j=1}^{n}\left(m_{1 j} \wedge m_{2 j}\right)}{\sum_{j=1}^{n}\left(m_{1 j} \vee m_{2 j}\right)}=\frac{\sum_{j=1}^{n} \sum_{k=1}^{q(j)}\left(a_{1 j k} \wedge a_{2 j k}+b_{1 j k} \wedge b_{2 j k}+\alpha_{1 j k} \wedge \alpha_{2 j k}\right)}{\sum_{j=1}^{n} \sum_{k=1}^{q(j)}\left(a_{1 j k} \vee a_{2 j k}+b_{1 j k} \vee b_{2 j k}+\alpha_{1 j k} \vee \alpha_{2 j k}\right)},
\end{aligned}
$$

where $\quad N\left(M_{1}, M_{2}\right)=\sum_{j=1}^{n} \sum_{k=1}^{q(j)}\left(a_{1 j k} a_{2 j k}+b_{1 j k} b_{2 j k}+\right.$ $\left.\alpha_{1 j k} \alpha_{2 j k}\right)$ is a correlation between $M_{1}$ and $M_{2}, N\left(M_{1}, M_{1}\right)=$ $\sum_{j=1}^{n} \sum_{k=1}^{q(j)}\left(a_{1 j k}^{2}+b_{1 j k}^{2}+\alpha_{1 j k}^{2}\right)$ and $N\left(M_{2}, M_{2}\right)=\sum_{j=1}^{n} \sum_{k=1}^{q(j)}$ $\left(a_{2 j k}^{2}+b_{2 j k}^{2}+\alpha_{2 j k}^{2}\right)$ are information energy of $M_{1}$ and $M_{2}$; then, the symbols " $\vee$ " and " $\wedge$ " are the maximum and minimum operations.

Regarding the properties of the correlation coefficient [17], the correlation coefficients $C_{p}\left(M_{1}, M_{2}\right)(p=1,2,3)$ also indicate the following proposition.

Proposition 1. The correlation coefficients $C_{p}\left(M_{1}, M_{2}\right)$ $(p=1,2,3)$ include the following properties:

(P1) $C_{p}\left(M_{1}, M_{2}\right)=1$ for $M_{1}=M_{2}$
(P2) $C_{p}\left(M_{1}, M_{2}\right)=C_{p}\left(M_{2}, M_{1}\right)$

(P3) $C p\left(M_{1}, M_{2}\right) \in[0,1]$

Proof. It is obvious that the verification of the properties (P1) and (P2) in Proposition 1 is straightforward. Hence, one only verifies the property (P3).

Based on the Cauchy-Schwarz inequality $\left(\sum_{j=1}^{n} y_{j}^{2}\right) \times$ $\left(\sum_{j=1}^{n} z_{j}^{2}\right) \geq\left(\sum_{j=1}^{n} y_{j} z_{j}\right)^{2}$ for any positive real numbers $y_{j}$ and $z_{j}(j=1,2, \ldots, n)$, there is the inequality $\sqrt{\sum_{j=1}^{n} y_{j}^{2}} \times \sqrt{\sum_{j=1}^{n} z_{j}^{2}} \geq \sum_{j=1}^{n} y_{j} z_{j}$. Hence, $C_{1}\left(M_{1}, M_{2}\right) \in[0$, 1] can hold based on the inequality. Then, it is obvious that there exist the following inequalities:

$$
\begin{aligned}
& \max \left(\sum_{j=1}^{n} \sum_{k=1}^{q(j)}\left(a_{1 j k}^{2}+b_{1 j k}^{2}+\alpha_{1 j k}^{2}\right), \sum_{j=1}^{n} \sum_{k=1}^{q(j)}\left(a_{2 j k}^{2}+b_{2 j k}^{2}+\alpha_{2 j k}^{2}\right)\right) \geq \sum_{j=1}^{n} \sum_{k=1}^{q(j)}\left(a_{1 j k} a_{2 j k}+b_{1 j k} b_{2 j k}+\alpha_{1 j k} \alpha_{2 j k}\right) \\
& \sum_{j=1}^{n} \sum_{k=1}^{q(j)}\left(a_{1 j k} \vee a_{2 j k}+b_{1 j k} \vee b_{2 j k}+\alpha_{1 j k} \vee \alpha_{2 j k}\right) \geq \sum_{j=1}^{n} \sum_{k=1}^{q(j)}\left(a_{1 j k} \wedge a_{2 j k}+b_{1 j k} \wedge b_{2 j k}+\alpha_{1 j k} \wedge \alpha_{2 j k}\right) .
\end{aligned}
$$

Thus, $C_{2}\left(M_{1}, M_{2}\right) \in[0,1]$ and $C_{3}\left(M_{1}, M_{2}\right) \in[0,1]$ can exist based on the above inequalities.

Therefore, the verification of Proposition 1 is completed.

If we consider the importance of MFCVs $m_{1 j}$ and $m_{2 j}(j=1$, $2, \ldots, n)$ in $M_{1}$ and $M_{2}$, one can give the weight $\beta_{j} \in[0,1]$ of $m_{1 j}$ and $m_{2 j}$ with $\sum_{j=1}^{n} \beta_{j}=1$. Thus, the weighted correlation coefficients of MFCSs $M_{1}$ and $M_{2}$ can be expressed by the following formulae:

$$
\begin{aligned}
& C_{w 1}\left(M_{1}, M_{2}\right)=\frac{N_{w}\left(M_{1}, M_{2}\right)}{\sqrt{N_{w}\left(M_{1}, M_{1}\right)} \sqrt{N_{w}\left(M_{2}, M_{2}\right)}}=\frac{\sum_{j=1}^{n} \beta_{j} \sum_{k=1}^{q(j)}\left(a_{1 j k} a_{2 j k}+b_{1 j k} b_{2 j k}+\alpha_{1 j k} \alpha_{2 j k}\right)}{\sqrt{\sum_{j=1}^{n} \beta_{j} \sum_{k=1}^{q(j)}\left(a_{1 j k}^{2}+b_{1 j k}^{2}+\alpha_{1 j k}^{2}\right)} \sqrt{\sum_{j=1}^{n} \beta_{j} \sum_{k=1}^{q(j)}\left(a_{2 j k}^{2}+b_{2 j k}^{2}+\alpha_{2 j k}^{2}\right)}}, \\
& C_{w 2}\left(M_{1}, M_{2}\right)=\frac{N_{w}\left(M_{1}, M_{2}\right)}{\max \left(N_{w}\left(M_{1}, M_{1}\right), N_{w}\left(M_{2}, M_{2}\right)\right)}=\frac{\sum_{j=1}^{n} \beta_{j} \sum_{k=1}^{q(j)}\left(a_{1 j k} a_{2 j k}+b_{1 j k} b_{2 j k}+\alpha_{1 j k} \alpha_{2 j k}\right)}{\max \left(\sum_{j=1}^{n} \beta_{j} \sum_{k=1}^{q(j)}\left(a_{1 j k}^{2}+b_{1 j k}^{2}+\alpha_{1 j k}^{2}\right), \sum_{j=1}^{n} \beta_{j} \sum_{k=1}^{q(j)}\left(a_{2 j k}^{2}+b_{2 j k}^{2}+\alpha_{2 j k}^{2}\right)\right)}, \\
& C_{w 3}\left(M_{1}, M_{2}\right)=\frac{\sum_{j=1}^{n} \beta_{j}\left(m_{1 j} \wedge m_{2 j}\right)}{\sum_{j=1}^{n} \beta_{j}\left(m_{1 j} \vee m_{2 j}\right)}=\frac{\sum_{j=1}^{n} \beta_{j} \sum_{k=1}^{q(j)}\left(a_{1 j k} \wedge a_{2 j k}+b_{1 j k} \wedge b_{2 j k}+\alpha_{1 j k} \wedge \alpha_{2 j k}\right)}{\sum_{j=1}^{n} \beta_{j} \sum_{k=1}^{q(j)}\left(a_{1 j k} \vee a_{2 j k}+b_{1 j k} \vee b_{2 j k}+\alpha_{1 j k} \vee \alpha_{2 j k}\right)}
\end{aligned}
$$


Similarly, the weighted correlation coefficients $C_{\mathrm{wp}}\left(M_{1}\right.$, $\left.M_{2}\right)(p=1,2,3)$ also indicate the following proposition.

Proposition 2. The weighted correlation coefficients $C_{w p}$ $\left(M_{1}, M_{2}\right)(p=1,2,3)$ contain the following properties:
(P1) $C_{w p}\left(M_{1}, M_{2}\right)=1$ for $M_{1}=M_{2}$
(P2) $C_{w p}\left(M_{1}, M_{2}\right)=C_{w p}\left(M_{2}, M_{1}\right)$
(P3) $C_{w p}\left(M_{1}, M_{2}\right) \in[0,1]$

Similar to the verification of Proposition 1, the verification of Proposition 2 is straightforward. Hence, it is not repeated here.

\section{Remark 1}

(i) When there exist all $\left[a_{1 j k}, b_{1 j k}\right]=\left[a_{2 j k}, b_{2 j k}\right]=[0,0]$ $(k=1,2, \ldots, q(j) ; j=1,2, \ldots, n)$ in $M_{1}$ and $M_{2}$, the weighted correlation coefficients of equations (7)-(9) are reduced to the following weighted correlation coefficients of MFSs:

$$
\begin{aligned}
& C_{w 4}\left(M_{1}, M_{2}\right)=\frac{N_{w}\left(M_{1}, M_{2}\right)}{\sqrt{N_{w}\left(M_{1}, M_{1}\right)} \sqrt{N_{w}\left(M_{2}, M_{2}\right)}}=\frac{\sum_{j=1}^{n} \beta_{j} \sum_{k=1}^{q(j)} \alpha_{1 j k} \alpha_{2 j k}}{\sqrt{\sum_{j=1}^{n} \beta_{j} \sum_{k=1}^{q(j)} \alpha_{1 j k}^{2}} \sqrt{\sum_{j=1}^{n} \beta_{j} \sum_{k=1}^{q(j)} \alpha_{2 j k}^{2}}}, \\
& C_{w 5}\left(M_{1}, M_{2}\right)=\frac{N_{w}\left(M_{1}, M_{2}\right)}{\max \left(N_{w}\left(M_{1}, M_{1}\right), N_{w}\left(M_{2}, M_{2}\right)\right)}=\frac{\sum_{j=1}^{n} \beta_{j} \sum_{k=1}^{q(j)} \alpha_{1 j k} \alpha_{2 j k}}{\max \left(\sum_{j=1}^{n} \beta_{j} \sum_{k=1}^{q(j)} \alpha_{1 j k}^{2}, \sum_{j=1}^{n} \beta_{j} \sum_{k=1}^{q(j)} \alpha_{2 j k}^{2}\right)}, \\
& C_{w 6}\left(M_{1}, M_{2}\right)=\frac{\sum_{j=1}^{n} \beta_{j}\left(m_{1 j} \wedge m_{2 j}\right)}{\sum_{j=1}^{n} \beta_{j}\left(m_{1 j} \vee m_{2 j}\right)}=\frac{\sum_{j=1}^{n} \beta_{j} \sum_{k=1}^{q(j)}\left(\alpha_{1 j k} \wedge \alpha_{2 j k}\right)}{\sum_{j=1}^{n} \beta_{j} \sum_{k=1}^{q(j)}\left(\alpha_{1 j k} \vee \alpha_{2 j k}\right)} .
\end{aligned}
$$

(ii) When there exist all $\alpha_{1 j k}=\alpha_{2 j k}=0(k=1,2, \ldots, q(j)$; $j=1,2, \ldots, n)$, the weighted correlation coefficients of equations (7)-(9) are reduced to the following weighted correlation coefficients of MIVFSs:

$$
\begin{aligned}
& C_{w 7}\left(M_{1}, M_{2}\right)=\frac{N_{w}\left(M_{1}, M_{2}\right)}{\sqrt{N_{w}\left(M_{1}, M_{1}\right)} \sqrt{N_{w}\left(M_{2}, M_{2}\right)}}=\frac{\sum_{j=1}^{n} \beta_{j} \sum_{k=1}^{q(j)}\left(a_{1 j k} a_{2 j k}+b_{1 j k} b_{2 j k}\right)}{\sqrt{\sum_{j=1}^{n} \beta_{j} \sum_{k=1}^{q(j)}\left(a_{1 j k}^{2}+b_{1 j k}^{2}\right)} \sqrt{\sum_{j=1}^{n} \beta_{j} \sum_{k=1}^{q(j)}\left(a_{2 j k}^{2}+b_{2 j k}^{2}\right)}}, \\
& C_{w 8}\left(M_{1}, M_{2}\right)=\frac{N_{w}\left(M_{1}, M_{2}\right)}{\max \left(N_{w}\left(M_{1}, M_{1}\right), N_{w}\left(M_{2}, M_{2}\right)\right)}=\frac{\sum_{j=1}^{n} \beta_{j} \sum_{k=1}^{q(j)}\left(a_{1 j k} a_{2 j k}+b_{1 j k} b_{2 j k}\right)}{\max \left(\sum_{j=1}^{n} \beta_{j} \sum_{k=1}^{q(j)}\left(a_{1 j k}^{2}+b_{1 j k}^{2}\right), \sum_{j=1}^{n} \beta_{j} \sum_{k=1}^{q(j)}\left(a_{2 j k}^{2}+b_{2 j k}^{2}\right)\right)}, \\
& C_{w 9}\left(M_{1}, M_{2}\right)=\frac{\sum_{j=1}^{n} \beta_{j}\left(m_{1 j} \wedge m_{2 j}\right)}{\sum_{j=1}^{n} \beta_{j}\left(m_{1 j} \vee m_{2 j}\right)}=\frac{\sum_{j=1}^{n} \beta_{j} \sum_{k=1}^{q(j)}\left(a_{1 j k} \wedge a_{2 j k}+b_{1 j k} \wedge b_{2 j k}\right)}{\sum_{j=1}^{n} \beta_{j} \sum_{k=1}^{q(j)}\left(a_{1 j k} \vee a_{2 j k}+b_{1 j k} \vee b_{2 j k}\right)} .
\end{aligned}
$$

(iii) Since the above-weighted correlation coefficients of MFSs and MFCSs are special cases of the weighted correlation coefficients of MFCSs, the three weighted correlation coefficients of MFCSs are more general and more useful in actual applications.

\section{GDM Methods Using the Weighted Correlation Coefficients of MFCSs}

This section develops multicriteria GDM methods using the weighted correlation coefficients in the environment of MFCSs.

In the GDM process, there usually exists a set of alternatives $M=\left\{M_{1}, M_{2}, \ldots, M_{s}\right\}$ to be assessed by a set of criteria $E=\left\{e_{1}\right.$, $\left.e_{2}, \ldots, e_{n}\right\}$ regarding a multicriteria GDM problem. Then, a weight vector $\beta=\left(\beta_{1}, \beta_{2}, \ldots, \beta_{n}\right)$ is specified to consider the importance weights of various criteria $e_{k}(j=1,2, \ldots, n)$ in $E$. Due to certain and uncertain situations of decision-makers' cognition regarding the different criteria, the satisfactory assessment values of the alternatives $M_{j}(j=1,2, \ldots, s)$ over the criteria $e_{j}(j=1,2, \ldots, n)$ are given by MFCVs $m_{i j}=<\left(\left[a_{i j}, a_{i j 1}\right]\right.$, $\left.\alpha_{i j 1}\right),\left(\left[a_{i j 2}, a_{i j 2}\right], \alpha_{i j 2}\right), \ldots,\left(\left[a_{i j q(j)}, a_{i j q(j)}\right], \alpha_{i j q(j)}\right)>(i=1,2, \ldots, s$; $j=1,2, \ldots, n)$ for $\left[a_{i j k}, a_{i j k}\right] \subseteq[0,1]$ and $\alpha_{i j k} \in[0,1](k=1,2, \ldots$, $q(j))$. Thus, all the assessed MFCVs can be constructed as the decision matrix of MFCSs $M=\left(m_{i j}\right)_{s \times n}$.

In the multicriteria GDM problem with the information of MFCSs, the decision algorithm of the multicriteria GDM methods is presented by the following steps: 
Step 1: regarding the decision matrix of MFCSs $M=\left(m_{i j}\right)_{s \times n}$, one first yields an ideal alternative $M^{*}=$ $\left\{m_{1}^{*}, m_{2}^{*}, \ldots, m_{n}^{*}\right\}$ by the following formula:

$$
\begin{aligned}
& m_{j}^{*}=\left\langle\left(\left[a_{j 1}^{*}, b_{j 1}^{*}\right], \alpha_{j 1}^{*}\right),\left(\left[a_{j 2}^{*}, b_{j 2}^{*}\right], \alpha_{j 2}^{*}\right), \ldots,\left(\left[a_{j q(j)}^{*}, b_{j q(j)}^{*}\right], \alpha_{j q(j)}^{*}\right)\right\rangle, \\
& \left(\left[\begin{array}{c}
\max \left(\max _{i}\left(a_{i j 1}\right), \max _{i}\left(a_{i j 2}\right), \ldots, \max _{i}\left(a_{i j q(j)}\right)\right), \\
\max \left(\max _{i}\left(b_{i j 1}\right), \max _{i}\left(b_{i j 2}\right), \ldots, \max _{i}\left(b_{i j q(j)}\right)\right)
\end{array}\right], \max \left(\max _{i}\left(\alpha_{i j 1}\right), \max _{i}\left(\alpha_{i j 2}\right), \ldots, \max _{i}\left(\alpha_{i j q(j)}\right)\right)\right), \\
& =\left\langle\left(\left[\begin{array}{c}
\max \left(\max _{i}\left(a_{i j 1}\right), \max _{i}\left(a_{i j 2}\right), \ldots, \max _{i}\left(a_{i j q(j)}\right)\right), \\
\max \left(\max _{i}\left(b_{i j 1}\right), \max _{i}\left(b_{i j 2}\right), \ldots, \max _{i}\left(b_{i j q(j)}\right)\right)
\end{array}\right], \max \left(\max _{i}\left(\alpha_{i j 1}\right), \max _{i}\left(\alpha_{i j 2}\right), \ldots, \max _{i}\left(\alpha_{i j q(j)}\right)\right)\right),\right\rangle, \\
& \left(\left[\begin{array}{c}
\max \left(\max _{i}\left(a_{i j 1}\right), \max _{i}\left(a_{i j 2}\right), \ldots, \max _{i}\left(a_{i j q(j)}\right)\right), \\
\max \left(\max _{i}\left(b_{i j 1}\right), \max _{i}\left(b_{i j 2}\right), \ldots, \max _{i}\left(b_{i j q(j)}\right)\right)
\end{array}\right], \max \left(\max _{i}\left(\alpha_{i j 1}\right), \max _{i}\left(\alpha_{i j 2}\right), \ldots, \max _{i}\left(\alpha_{i j q(j)}\right)\right)\right)
\end{aligned}
$$

Step 2: the values of the weighted correlation coefficient are obtained by using one of the three weighted correlation coefficients $C_{\mathrm{wp}}\left(M_{i}, M^{*}\right)(p=1,2,3 ; i=1,2$, $\ldots, s)$ :

$$
\begin{aligned}
C_{w 1}\left(M_{i}, M^{*}\right) & =\frac{\sum_{j=1}^{n} \beta_{j} \sum_{k=1}^{q(j)}\left(a_{i j k} a_{j k}^{*}+b_{i j} b_{j k}^{*}+\alpha_{i j} \alpha_{j k}^{*}\right)}{\sqrt{\sum_{j=1}^{n} \beta_{j} \sum_{k=1}^{q(j)}\left(a_{1 j k}^{2}+b_{1 j k}^{2}+\alpha_{1 j k}^{2}\right)} \sqrt{\sum_{j=1}^{n} \beta_{j} \sum_{k=1}^{q(j)}\left[\left(a_{j k}^{*}\right)^{2}+\left(b_{j k}^{*}\right)^{2}+\left(\alpha_{j k}^{*}\right)^{2}\right]}}, \\
C_{w 2}\left(M_{i}, M^{*}\right) & =\frac{\sum_{j=1}^{n} \beta_{j} \sum_{k=1}^{q(j)}\left(a_{i j k} a_{j k}^{*}+b_{i j k} b_{j k}^{*}+\alpha_{i j k} \alpha_{j k}^{*}\right)}{\sum_{j=1}^{n} \beta_{j} \sum_{k=1}^{q(j)}\left[\left(a_{j k}^{*}\right)^{2}+\left(b_{j k}^{*}\right)^{2}+\left(\alpha_{j k}^{*}\right)^{2}\right]}, \\
C_{w 3}\left(M_{i}, M^{*}\right) & =\frac{\sum_{j=1}^{n} \beta_{j} \sum_{k=1}^{q(j)}\left(a_{i j k}+b_{i j k}+\alpha_{i j k}\right)}{\sum_{j=1}^{n} \beta_{j} \sum_{k=1}^{q(j)}\left(a_{j k}^{*}+b_{j k}^{*}+\alpha_{j k}^{*}\right)} .
\end{aligned}
$$

Step 3: ranking of the alternatives is given in descending order of the values of the weighted correlation coefficient and the best one is selected.

Step 4: end.

\section{Illustrative Example and Comparative Analysis}

6.1. GDM Example on the Selection Problem of Equipment Suppliers. A manufacturing company wants to enhance the competitiveness of products and to enlarge the production capacity, the company plans to replace an existing equipment supplier to improve the product quality. Thus, the decision department has to select four potential suppliers preliminarily as a set of four alternatives $M=\left\{M_{1}, M_{2}, M_{3}, M_{4}\right\}$. Then, the company must make a decision by the suitable assessment of the four alternatives over the requirements of three criteria: cost $\left(e_{1}\right)$, quality $\left(e_{2}\right)$, and supplier's reputation $\left(e_{3}\right)$. The importance of the three criteria is taken into account by the weight vector $\beta=(0.35,0.4,0.25)$. Thus, the company invites three experts/decision-makers to give the assessment values of the four alternatives over the three criteria based on the partial certain and partial uncertain judgments of their cognition, which are presented in the form of MFCVs $m_{i j}=<\left(\left[a_{i j 1}, b_{i j 1}\right]\right.$, $\left.\alpha_{i j 1}\right),\left(\left[a_{i j 2}, b_{i j 2}\right], \alpha_{1 j 2}\right),\left(\left[a_{i j 3}, b_{i j 3}\right], \alpha_{i j 3}\right)>$ for $\left[a_{i j k}, b_{i j k}\right] \subseteq[0,1]$ and $\alpha_{i j k} \in[0,1](j, k=1,2,3 ; i=1,2,3,4)$. Thus, all the assessed MFCVs can be constructed as the decision matrix of MFCSs $M=\left(m_{i j}\right)_{4 \times 3}$ in Table 1 .

By applying equation (16) for the decision matrix of Table 1, we first give the ideal alternative:

$M^{*}=\left\{m_{1}^{*}, m_{2}^{*}, m_{3}^{*}\right\}=\{<([0.8,0.9], 0.9),([0.8,0.9], 0.9)$, $([0.8,0.9], 0.9)>,<([0.8,0.9], 0.9),([0.8,0.9], 0.9),([0.8,0.9]$, $0.9)>,<([0.8,0.95], 0.9),([0.8,0.95], 0.9),([0.8,0.95], 0.9)>\}$. 
TABLE 1: The decision matrix of MFCSs.

\begin{tabular}{|c|c|c|c|}
\hline & $e_{1}$ & $e_{2}$ & $e_{3}$ \\
\hline$M_{1}$ & $\begin{array}{c}<([0.7,0.8], 0.7),([0.6,0.8], 0.7),([0.6 \\
0.7], 0.6)>\end{array}$ & $\begin{array}{c}<([0.7,0.9], 0.8),([0.7,0.8], 0.7),([0.7,0.8]), \\
0.7)>\end{array}$ & $\begin{array}{c}<([0.8,0.9], 0.9),([0.8,0.9]), 0.8),([0.7 \\
0.8], 0.7)>\end{array}$ \\
\hline$M_{2}$ & $\begin{array}{c}<([0.8,0.9], 0.8),([0.7,0.9]), 0.8),([0.7, \\
0.8], 0.7)>\end{array}$ & $\begin{array}{c}<([0.8,0.9], 0.9),([0.8,0.9], 0.8),([0.7,0.9]) \\
0.7)>\end{array}$ & $\begin{array}{c}<([0.7,0.9], 0.7),([0.7,0.8]), 0.7),([0.7 \\
0.7], 0.7)>\end{array}$ \\
\hline$M_{3}$ & $\begin{array}{c}<([0.7,0.9], 0.8),([0.7,0.8]), 0.7),([0.7, \\
0.8], 0.7)>\end{array}$ & $\begin{array}{c}<([0.7,0.85], 0.75),([0.7,0.8], 0.7),([0.6 \\
0.8]), 0.7)>\end{array}$ & $\begin{array}{c}<([0.7,0.9], 0.8),([0.7,0.8]), 0.7),([0.7 \\
0.8], 0.7)>\end{array}$ \\
\hline$M_{4}$ & $\begin{array}{c}<([0.8,0.9], 0.9),([0.8,0.9]), 0.8),([0.7, \\
0.8], 0.7)>\end{array}$ & 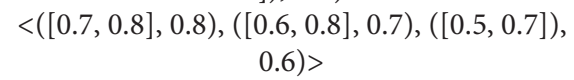 & $\begin{array}{c}<([0.8,0.95], 0.9),([0.8,0.9]), 0.8),([0.7, \\
0.9], 0.8)>\end{array}$ \\
\hline
\end{tabular}

By using one of equations (17)-(19), we obtain the values of $C_{w p}\left(M_{i}, M^{*}\right)(p=1,2,3 ; i=1,2,3,4)$. Then, their decision results are indicated in Table 2 .

In the decision results of Table 2, the ranking orders based on the two weighted correlation coefficients $C_{w 2}\left(M_{i}\right.$, $\left.M^{*}\right)$ and $C_{w 3}\left(M_{i}, M^{*}\right)$ are identical in the GDM example, but different from the ranking order based on the weighted correlation coefficient $C_{w 1}\left(M_{i}, M^{*}\right)$. Then, the best alternative is $M_{2}$ or $M_{3}$. However, the different weighted correlation coefficients can affect the ranking orders of the four alternatives; then the final decision result depends on the weighted correlation coefficient used by decision-makers.
6.2. Comparative Analysis of GDM Methods. This part presents the comparative analysis of the developed GDM methods and the related methods to demonstrate the practicability and suitability of the developed GDM methods.

First, we only consider the multicriteria GDM problems in the environments of MFSs and MIVFSs as two special cases of the above illustrative example in the environment of MFCSs. Regarding the GDM problems of the two special cases, if there exist either all $\left[a_{i j k}, b_{i j k}\right]=[0,0]$ or all $\alpha_{i j k}=0(j$, $k=1,2,3$ and $i=1,2,3,4)$ in the decision matrix of MCFSs $M=\left(m_{i j}\right)_{4 \times 3}$, the decision matrix $M=\left(m_{i j}\right)_{4 \times 3}$ is reduced to the decision matrices of MFSs or MIVFSs:

$$
\begin{aligned}
& M_{\mathrm{MFS}}=\left[\begin{array}{l}
M_{\mathrm{MFS} 1} \\
M_{\mathrm{MFS} 2} \\
M_{\mathrm{MFS} 3} \\
M_{\mathrm{MFS} 4}
\end{array}\right]=\left[\begin{array}{ccc}
(0.7,0.7,0.6) & (0.8,0.7,0.7) & (0.9,0.8,0.7) \\
(0.8,0.8,0.7) & (0.9,0.8,0.7) & (0.7,0.7,0.7) \\
(0.7,0.7,0.6) & (0.75,0.7,0.7) & (0.8,0.7,0.7) \\
(0.9,0.8,0.7) & (0.8,0.7,0.6) & (0.9,0.8,0.8)
\end{array}\right] \\
& M_{\mathrm{MIVFS}}=\left[\begin{array}{c}
M_{\mathrm{MIVSS} 1} \\
M_{\mathrm{MIVFS} 2} \\
M_{\mathrm{MIVFS} 3} \\
M_{\mathrm{MIVFS}}
\end{array}\right] \\
& =\left[\begin{array}{lcc}
([0.7,0.8],[0.6,0.8],[0.6,0.7]) & ([0.7,0.9],[0.7,0.8],[0.7,0.8]) & ([0.8,0.9],[0.8,0.9],[0.7,0.8]) \\
([0.8,0.9],[0.7,0.9],[0.7,0.8]) & ([0.8,0.9],[0.8,0.9],[0.7,0.9]) & ([0.7,0.9],[0.7,0.8],[0.7,0.7]) \\
([0.7,0.8],[0.6,0.8],[0.6,0.7]) & ([0.7,0.85],[0.7,0.8],[0.6,0.8]) & ([0.7,0.9],[0.7,0.8],[0.7,0.8]) \\
([0.8,0.9],[0.8,0.9],[0.7,0.8]) & ([0.7,0.8],[0.6,0.8],[0.5,0.7]) & ([0.8,0.95],[0.8,0.9],[0.7,0.9])
\end{array}\right] .
\end{aligned}
$$

Based on the decision matrices of MFSs and MIVFSs, the two ideal alternatives are yielded in the following:

$$
\begin{aligned}
& M_{1}^{*}=\left\{m_{1}^{*}, m_{2}^{*}, m_{3}^{*}\right\}=\{(0.9,0.9,0.9),(0.9,0.9,0.9),(0.9, \\
& 0.9,0.9)\}, \\
& M_{2}^{*}=\left\{m_{1}^{*}, m_{2}^{*}, m_{3}^{*}\right\}=\{([0.8,0.9],[0.8,0.9],[0.8,0.9]), \\
& ([0.8,0.9],[0.8,0.9],[0.8,0.9]),([0.8,0.95],[0.8,0.95], \\
& [0.8,0.95])\} .
\end{aligned}
$$

Thus, by applying equations (10)-(15), we obtain all decision results of the weighted correlation coefficients of MFSs and MIVFSs, which are shown in Table 3.

In the decision results of Table 3, the ranking orders of the four alternatives based on different weighted correlation coefficients under different environments of MFSs and MIVFSs indicate different ranking results, and then the best alternative is $M_{2}$ or $M_{3}$ or $M_{4}$.

Regarding the decision results of Tables 2 and 3, it is clear that the developed multifuzzy cubic GDM methods contain the multifuzzy and multi-interval-valued fuzzy GDM methods under the specifical environments of MFSs and MIVFSs. However, their ranking orders and the best ones show the partial same and partial difference based on different weighted correlation coefficients under different environments of MFCSs, MFSs, and MIVFSs, which show that either different weighted correlation coefficients or different information environments can impact on the ranking orders 
TABle 2: Decision results of the weighted correlation coefficients between $M_{i}$ and $M^{*}$.

\begin{tabular}{lccc}
\hline Weighted correlation coefficient & Value of weighted correlation coefficient & Ranking & The best one \\
\hline$C_{w 1}\left(M_{i}, M^{*}\right)$ & $0.9951,0.9954,0.9966,0.9929$ & $M_{3}>M_{2}>M_{1}>M_{4}$ & $M_{3}$ \\
$C_{w 2}\left(M_{i}, M^{*}\right)$ & $0.8567,0.9043,0.8309,0.8840$ & $M_{2}>M_{4}>M_{1}>M_{3}$ & $M_{2}$ \\
$C_{w 3}\left(M_{i}, M^{*}\right)$ & $0.8568,0.9053,0.8306,0.8833$ & $M_{2}>M_{4}>M_{1}>M_{3}$ & $M_{2}$ \\
\hline
\end{tabular}

TABLE 3: Decision results of the weighted correlation coefficients between $M_{\mathrm{MFS} i}$ and $M_{1}^{*}$ and between $M_{\mathrm{MIVFS} i}$ and $M_{2}^{*}$.

\begin{tabular}{lclc}
\hline Weighted correlation coefficient & Value of weighted correlation coefficient & Ranking & The best one \\
\hline$C_{w 4}\left(M_{\mathrm{MFS} i}, M_{1}^{*}\right)$ & $0.9944,0.9957,0.9977,0.9926$ & $M_{3}>M_{2}>M_{1}>M_{4}$ & $M_{3}$ \\
$C_{w 5}\left(M_{\mathrm{MFS} i}, M_{1}^{*}\right)$ & $0.8074,0.8481,0.7815,0.8537$ & $M_{4}>M_{2}>M_{1}>M_{3}$ & $M_{4}$ \\
$C_{w 6}\left(M_{\mathrm{MFS} i}, M_{1}^{*}\right)$ & $0.8074,0.8481,0.7815,0.8537$ & $M_{4}>M_{2}>M_{1}>M_{3}$ & $M_{4}$ \\
$C_{w 7}\left(M_{\mathrm{MIVFS}}, M_{2}^{*}\right)$ & $0.9968,0.9967,0.9976,0.9935$ & $M_{3}>M_{1}>M_{2}>M_{4}$ & $M_{3}$ \\
$C_{w 8}\left(M_{\mathrm{MIVFS} i}, M_{2}^{*}\right)$ & $0.8838,0.9352,0.8581,0.9006$ & $M_{2}>M_{4}>M_{1}>M_{3}$ & $M_{2}$ \\
$C_{w 9}\left(M_{\mathrm{MIVFS} i}, M_{2}^{*}\right)$ & $0.8827,0.9353,0.8564,0.8988$ & $M_{2}>M_{4}>M_{1}>M_{3}$ & $M_{2}$ \\
\hline
\end{tabular}

of alternatives in the GDM example. Since MFCSs consist of both MFSs and MIVFSs, the multifuzzy and multi-intervalvalued fuzzy GDM methods introduced in this study are only special cases of the multifuzzy cubic DM methods. Therefore, the developed multifuzzy cubic DM methods are more general and more reasonable in the information expressions and decision usability, which show their highlighting advantages in the setting of MFCSs.

Since hesitant cubic FSs [26, 27], CHFSs [28-30], CHNNs [31], FCCNs [32], and cubic IFSs [33-35] cannot express the MFCS information, their decision-making methods [26-35] cannot also carry out the GDM problems in the setting of MFCSs. In the situations, it is obvious that the developed multifuzzy cubic GDM methods also indicate their superiority and usability in the setting of MFCSs.

\section{Conclusion}

Regarding the hybrid expression problem of MFS and MIVFS in the multifuzzy setting of uncertainty and certainty, this paper presented a MFCS concept as the extension of FCS in order to adequately depict the opinion of several experts/decision-makers by using a union/sequence of the same and/or different FCVs. Then, we proposed three correlation coefficients of MFCSs and after that introduced the correlation coefficients of MFSs and MIVFSs as the special cases of the three correlation coefficients of MFCSs. Further, multicriteria GDM methods using the three weighted correlation coefficients of MFCSs were developed under the environment of MFCSs, which contain the multifuzzy and multi-interval-valued fuzzy GDM methods. Lastly, these multicriteria GDM methods were applied in an illustrative example on the selection problem of equipment suppliers; then their decision results and comparative analysis indicated that the developed GDM methods are more practicable and effective and reflected that either different correlation coefficients or different information expressions can impact on the ranking orders of alternatives.

As future work, we shall extend this original study to clustering analysis, pattern recognition, and image processing under the environment of MFCSs and further develop new similarity measures of MFCSs for GDM, medical diagnosis, pattern recognition, and so on.

\section{Data Availability}

No data were used to support the results in this study.

\section{Conflicts of Interest}

The authors declare no conflicts of interest.

\section{References}

[1] L. A. Zadeh, "Fuzzy sets," Information and Control, vol. 8, no. 3, pp. 338-353, 1965.

[2] S. K. Pal and R. A. King, "On edge detection of X-ray images using fuzzy sets," IEEE Transactions on Pattern Analysis and Machine Intelligence, vol. PAMI-5, no. 1, pp. 69-77, 1983.

[3] V. U. Nguyen, “Tender evaluation by fuzzy sets," Journal of Construction Engineering and Management, vol. 111, no. 3, pp. 231-243, 1985.

[4] R. R. Yager, "Database discovery using fuzzy sets," International Journal of Intelligent Systems, vol. 11, pp. 691-712, 1996.

[5] D.-A. Chiang and N. P. Lin, "Correlation of fuzzy sets," Fuzzy Sets and Systems, vol. 102, no. 2, pp. 221-226, 1999.

[6] G. E. Gürcanli and U. Müngen, "An occupational safety risk analysis method at construction sites using fuzzy sets," International Journal of Industrial Ergonomics, vol. 39, no. 2, pp. 371-387, 2009.

[7] M. Ansari, R. S. Hosseini, and M. Sharifi, "Evaluating and rating the performance of Qazvin municipalities, using the balanced scorecard (BSC) model, with fuzzy multi-criteria decision-making (FMCDM) approach," International Journal of Human-Computer Studies, vol. 3, no. 2, pp. 185-197, 2016.

[8] H. Barghi Jond, V. Nabiyev, and R. Benveniste, "Analysis of customer's expectations and satisfaction in Zanjan municipality using fuzzy multi-criteria decision making (FMCDM) approach," Journal of Optimization Industrial Engineering, vol. 10, no. 21, pp. 47-57, 2017.

[9] M. M. Pandey, D. Shukla, and A. Graham, "Evaluating the human performance factors of air traffic control in Thailand using fuzzy multi criteria decision making method," Journal of Air Transport Management, vol. 81, p. 101708, 2019. 
[10] B. Bostanc1 and N. Erdem, "Investigating the satisfaction of citizens in municipality services using fuzzy modeling," SocioEconomic Planning Sciences, vol. 69, p. 100754, 2020.

[11] R. R. Yager, "On the theory of bags," International Journal of General Systems, vol. 13, no. 1, pp. 23-37, 1986.

[12] S. Sebastian and T. V. Ramakrishnan, "Multi-fuzzy sets: an extension of fuzzy sets," Fuzzy Information and Engineering, vol. 3, no. 1, pp. 35-43, 2011.

[13] S. Miyamoto, "Fuzzy multisets and fuzzy clustering of documents," 2001.

[14] J.-P. Banâtre and D. Le Métayer, "Programming by multiset transformation," Communications of the ACM, vol. 36, no. 1, pp. 98-111, 1993.

[15] B. Li, "Fuzzy bags and application," Fuzzy Sets and Systems, vol. 34, pp. 67-71, 1999.

[16] S. Miyamoto, "Generalized bags, bag relations, and applications to data analysis and decision making," Modeling Decisions for Artificial Intelligence, vol. 5861, pp. 37-54, 2009.

[17] M. S. El-Azab, M. Shokry, and R. A. Abo khadra, "Correlation measure for fuzzy multisets," Journal of the Egyptian Mathematical Society, vol. 25, no. 3, pp. 263-267, 2017.

[18] M. B. Gorzałczany, "A method of inference in approximate reasoning based on interval valued fuzzy sets," Fuzzy Sets and Systems, vol. 21, no. 1, pp. 1-17, 1987.

[19] V. Kreinovich and S. Sriboonchitta, "For Multi-interval-valued fuzzy sets, centroid defuzzification is equivalent to defuzzifying its interval hull: a theorem," in Advances in Computational Intelligence, MICAI 2016, Lecture Notes in Computer Science, G. Sidorov and O. Herrera-Alcántara, Eds., Vol. 10061, Springer, Berlin, Germany, 2017.

[20] Y. B. Jun, C. S. Kim, and K. O. Yang, "Cubic sets," Annals of Fuzzy Mathematics and Informatics, vol. 4, no. 1, pp. 83-98, 2012.

[21] Y. B. Jun, S. T. Jung, and M. S. Kim, "Cubic subgroups," Annals of Fuzzy Mathematics and Informatics, vol. 2, no. 1, pp. 9-15, 2011.

[22] Y. B. Jun and A. Khan, "Cubic ideals in semigroups," Honam Mathematical Journal, vol. 35, no. 4, pp. 607-623, 2013.

[23] S. Vijayabalaji and S. Sivaramakrishnan, "A cubic set theoretical approach to linear space," Abstract and Applied Analysis, vol. 2015, pp. 1-8, Article ID 523129, 2015.

[24] J. M. Wang, W. H. Cui, and J. Ye, "Slope stability evaluation using tangent similarity measure of fuzzy cube sets," Soft Computing in Civil Engineering, vol. 3, no. 1, pp. 27-35, 2019.

[25] V. Torra, "Hesitant fuzzy sets," International Journal of Intelligent Systems, vol. 25, no. 6, pp. 529-539, 2010.

[26] Q. Khan, T. Mahmood, and F. Mehmood, "Cubic hesitant fuzzy sets and their applications to multi criteria decision making," International Journal of Algebra and Statistics, vol. 5, pp. 19-51, 2016.

[27] A. Fahmi and F. Amin, "Precursor selection for sol-gel synthesis of titanium carbide nanopowders by a new hesitant CUBIC fuzzy multi-attribute group decision-making model," New Mathematics and Natural Computation, vol. 15, no. 1, pp. 145-167, 2019.

[28] J. Fu, J. Ye, and W. Cui, "An evaluation method of risk grades for prostate cancer using similarity measure of cubic hesitant fuzzy sets," Journal of Biomedical Informatics, vol. 87, pp. 131-137, 2018.

[29] J. Fu, J. Ye, and W. Cui, "The Dice measure of cubic hesitant fuzzy sets and its initial evaluation method of benign prostatic hyperplasia symptoms," Scientific Reports, vol. 9, no. 1, p. 60, 2019.
[30] R. Yong, A. Zhu, and J. Ye, "Multiple attribute decision method using similarity measure of cubic hesitant fuzzy sets," Journal of Intelligent \& Fuzzy Systems, vol. 37, no. 1, pp. 1075-1083, 2019.

[31] J. Fu and J. Ye, "Similarity measure with indeterminate parameters regarding cubic hesitant neutrosophic numbers and its risk grade assessment approach for prostate cancer patients," Applied Intelligence, vol. 50, no. 7, pp. 2120-2131, 2020.

[32] J. Ye, S. Du, R. Yong, and F. Zhang, "Weighted aggregation operators of fuzzy credibility cubic numbers and their decision making strategy for slope design schemes," Current Chinese Computer Science, vol. 1, 2020.

[33] H. Garg and G. Kaur, "Cubic intuitionistic fuzzy sets and its fundamental properties," Journal of Multiple-Valued Logic and Soft Computing, vol. 33, no. 6, pp. 507-537, 2019.

[34] H. Garg and G. Kaur, "TOPSIS based on nonlinear-programming methodology for solving decision-making problems under cubic intuitionistic fuzzy set environment," Computational and Applied Mathematics, vol. 38, no. 114, 2019.

[35] H. Garg and G. Kaur, "Extended TOPSIS method for multicriteria group decision-making problems under cubic intuitionistic fuzzy environment," Scientia Iravnica, vol. 27, no. 1, pp. 396-410, 2020.

[36] M. Akram, W. A. Dudek, and F. Ilyas, "Group decision making based on Pythagorean fuzzy TOPSIS method," International Journal of Intelligent System, vol. 34, no. 156, pp. 1455-1475, 2019.

[37] Z. Mua, S. Zeng, and P. Wang, "Novel approach to multiattribute group decision-making based on interval-valued Pythagorean fuzzy power Maclaurin symmetric mean operator," Computers \& Industrial Engineering, vol. 2020, 2020.

[38] S. Zeng, Y. Hu, T. Balezentis, and D. Streimikiene, "A multicriteria sustainable supplier selection framework based on neutrosophic fuzzy data and entropy weighting," Sustainable Development, vol. 28, no. 5, pp. 1431-1440, 2020. 